\title{
On the prospective of polynuclear complexes with acetylenedithiolate bridging units
}

Wolfram W. Seidel,* Matthias J. Meel, Udo Radius, Markus Schaffrath and Tania Pape

\section{Supporting Information}

$\underline{\text { Arrangement of K-3c in tetrameric units in the crystal }}$

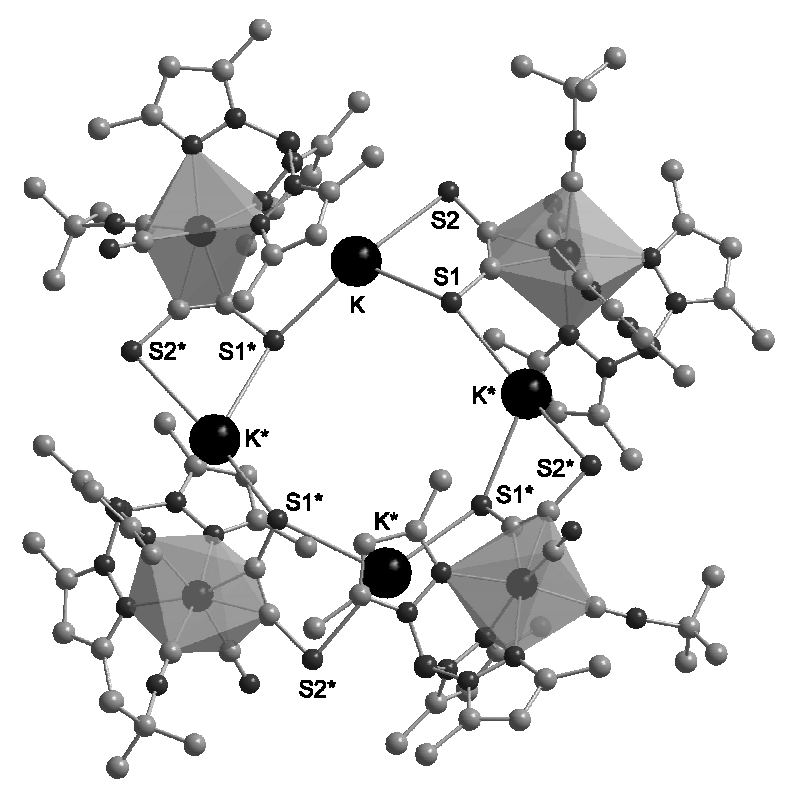

Figure S1. Ball and stick representation of $(\mathrm{K}-3 \mathbf{c})_{4}$.

\section{$\underline{\text { UV-vis spectroscopic data of polynuclear complexes }}$}

Table S1. Maxima in the electronic spectra of complexes in $\mathrm{CH}_{2} \mathrm{Cl}_{2}$ solution.

\begin{tabular}{cl}
\hline complex & $\lambda_{\max } / \mathrm{nm}\left(\varepsilon / 10^{3} \cdot \mathrm{M}^{-1} \cdot \mathrm{cm}^{-1}\right)$ \\
\hline $\mathbf{4 a}$ & $343(12.0), 452(3.3), 618(11.2)$ \\
$\mathbf{4 a}^{+}$ & $331(10.0), 445(5.5), 588(7.0), 758(2.0)$ \\
$\mathbf{4 b}$ & $318(21.0), 571(11.5)$ \\
$\mathbf{4 b}$ & $323(16.0), 442(7.5), 590(14.5)$ \\
$\mathbf{5 a}$ & $311(29.5), 374(42.0), 649(19.0)$ \\
$\mathbf{5 b}$ & $341(38.5), 501(41.0)$ \\
$\mathbf{5 c}$ & $334(23.7), 376(9.9), 468(\mathrm{sh}, 7.9), 599(43.0)$ \\
$\mathbf{6 a}$ & $352(21.5), 512(14.5)$ \\
$\mathbf{6 b}$ & $345(18.5), 605(16.0)$
\end{tabular}




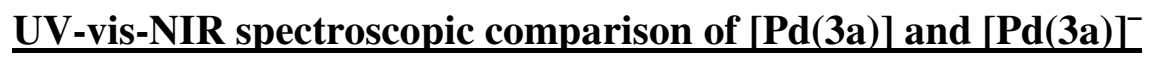
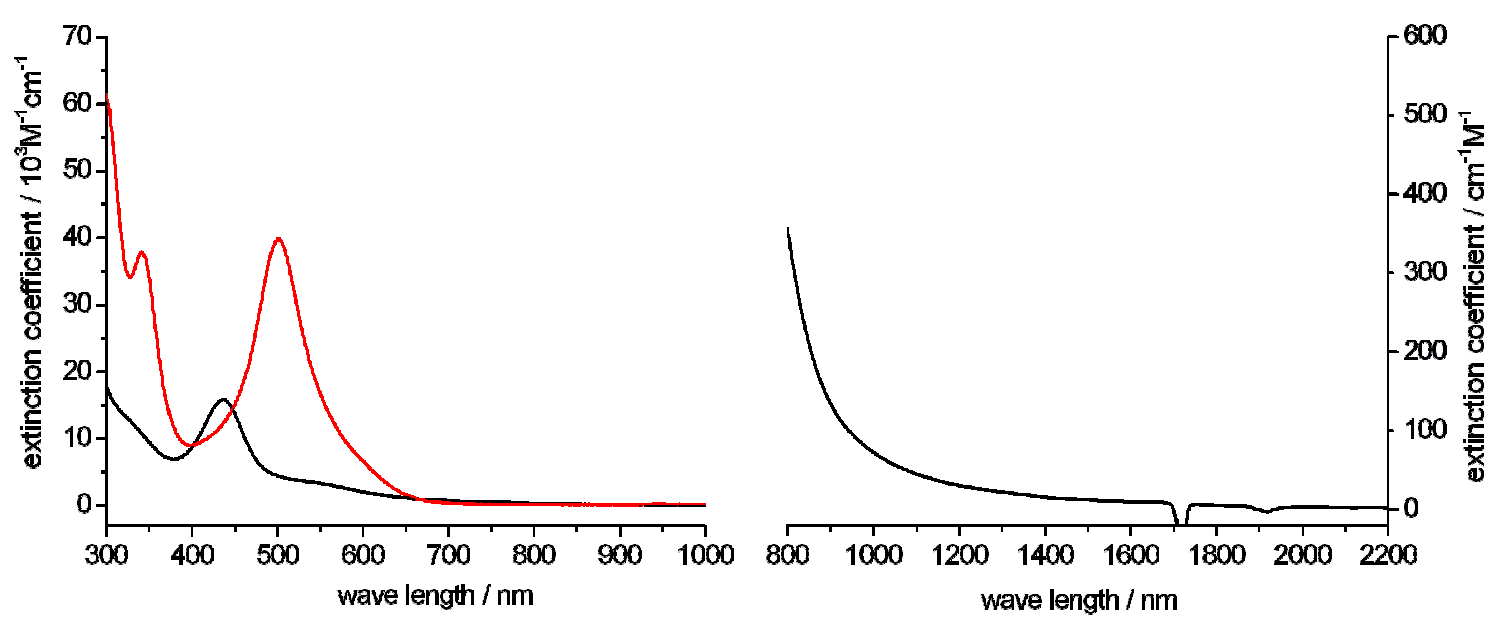

Figure S2. Electronic spectra of $[\operatorname{Pd}(\mathbf{3 a})]\left(\right.$ red) and $[\operatorname{Pd}(\mathbf{3 a})]^{-}$(black).

\section{$\underline{\text { EPR spectroscopic data }}$}
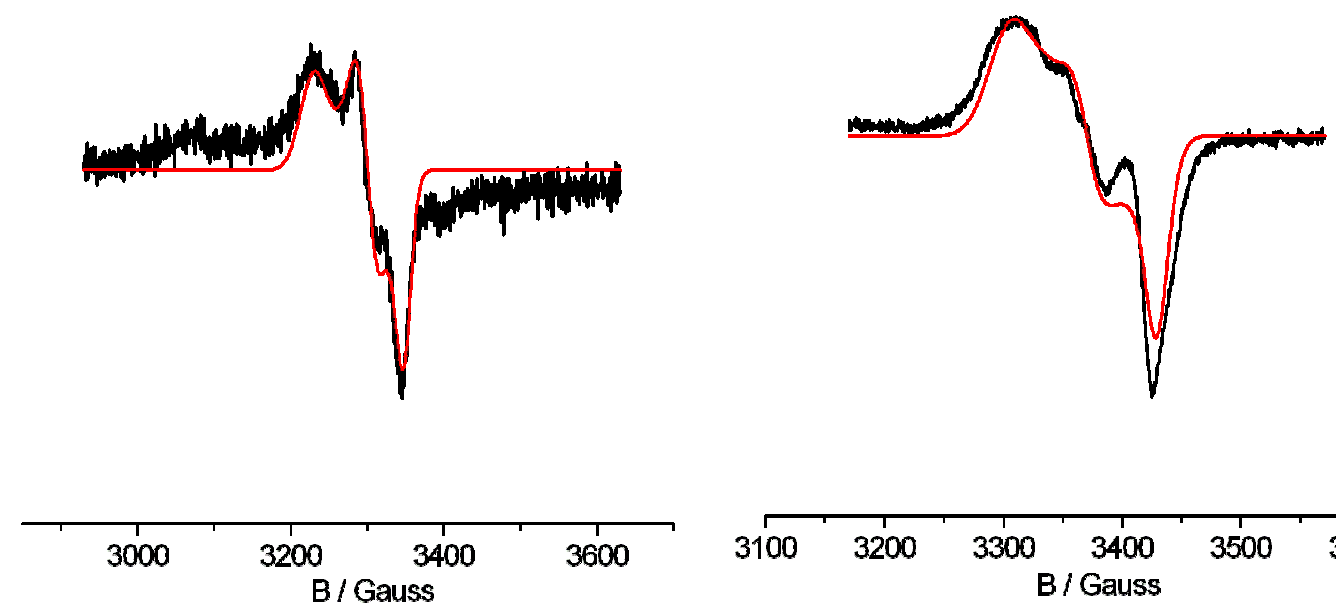

Figure S 3. EPR spectra of $[\operatorname{Pd}(\mathbf{3 a})]^{-}$(left) and $[\operatorname{Pt}(\mathbf{3 a})]^{-}$(right) in a frozen THF solution at $-77 \mathrm{~K}$; black: observed, red simulated.

Simulation parameters:

\begin{tabular}{lcc}
\hline & $g_{\mathrm{i}}$ & $a_{\mathrm{i}}\left({ }^{195} \mathrm{Pt}\right) /$ Gauss \\
\hline$[\mathrm{Pd}(\mathbf{3 a})]^{-}$ & $1.972 / 2.000 / 2.044$ & \\
{$[\mathrm{Pt}(\mathbf{3 a})]^{-}$} & $1.966 / 2.000 / 2.039$ & $18 / 30 / 18$
\end{tabular}


Table S2. Metric parameter of the central dimetalla acetylenedithiolate unit in the calculated structures of complexes $\mathbf{4 a} / \mathbf{4} \mathbf{a}^{\prime}, \mathbf{4 b} / \mathbf{4} \mathbf{b}^{6}$ and their cations.

\begin{tabular}{|c|c|c|c|c|c|c|c|c|}
\hline $\mathrm{BD} / \mathrm{BA}$ & $4 a^{\prime}$ & 4b' & $4 a$ & $4 b$ & $4 a^{9+}$ & $4 b^{++}$ & $4 a^{+}$ & $4 b^{+}$ \\
\hline W-C1 & 2.1072 & 2.1035 & 2.1130 & 2.1060 & 2.0894 & 2.0907 & 2.0930 & 2.0923 \\
\hline $\mathrm{W}-\mathrm{C} 2$ & 2.0775 & 2.0693 & 2.0871 & 2.0768 & 2.0618 & 2.0618 & 2.0771 & 2.0764 \\
\hline $\mathrm{Ru}-\mathrm{S} 1$ & 2.3988 & 2.4075 & 2.4131 & 2.4136 & 2.3879 & 2.3996 & 2.3773 & 2.3844 \\
\hline $\mathrm{Ru}-\mathrm{S} 2$ & 2.3981 & 2.4176 & 2.4061 & 2.4175 & 2.3582 & 2.3824 & 2.3618 & 2.3740 \\
\hline $\mathrm{C} 1-\mathrm{C} 2$ & 1.3806 & 1.3828 & 1.3827 & 1.3834 & 1.3839 & 1.3848 & 1.3809 & 1.3795 \\
\hline $\mathrm{C} 1-\mathrm{S} 1$ & 1.6882 & 1.6836 & 1.6892 & 1.6862 & 1.6932 & 1.6887 & 1.6982 & 1.6940 \\
\hline $\mathrm{C} 2-\mathrm{S} 2$ & 1.6919 & 1.6884 & 1.6949 & 1.6944 & 1.7007 & 1.6955 & 1.7016 & 1.7010 \\
\hline $\mathrm{S} 1-\mathrm{S} 2$ & 3.2640 & 3.2974 & 3.2683 & 3.3008 & 3.3104 & 3.3740 & 3.2879 & 3.3154 \\
\hline $\begin{array}{l}\text { C1-C2- } \\
\text { S2 }\end{array}$ & 122.388 & 123.167 & 121.496 & 122.157 & 123.266 & 124.128 & 121.786 & 122.350 \\
\hline $\begin{array}{l}\text { C2-C1- } \\
\text { S1 }\end{array}$ & 125.340 & 126.031 & 126.193 & 126.924 & 125.913 & 126.750 & 126.423 & 127.168 \\
\hline $\mathrm{S} 1-\mathrm{Ru}-\mathrm{S} 2$ & 85.756 & 86.218 & 85.403 & 86.197 & 88.451 & 88.842 & 87.862 & 88.333 \\
\hline
\end{tabular}

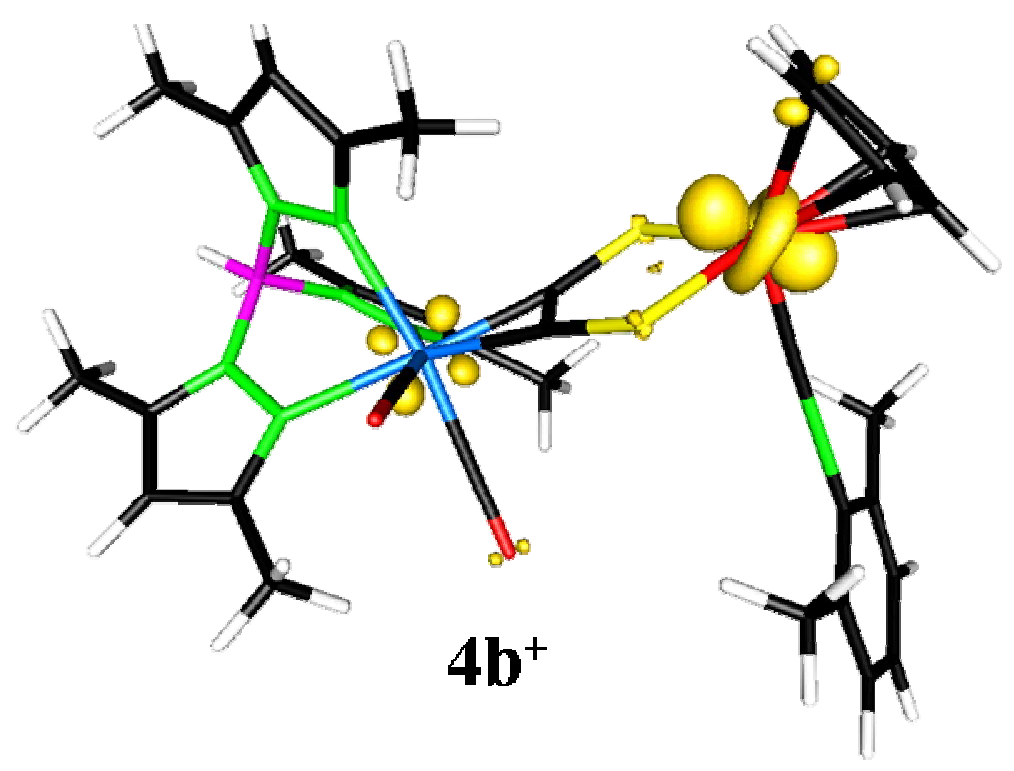

Figure S4. Spin density calculated for $\mathbf{4} \mathbf{b}^{+}$. 